\title{
Cyclophilin A from Schistosoma japonicum promotes a Th2 response in mice
}

\author{
Jinghui $\mathrm{Li}^{1 \dagger}$, Wenjia Zhuang ${ }^{1 \dagger}$, Li Cong ${ }^{1}$, Wenjun Shi ${ }^{2}$ Xingyan Cai ${ }^{1}$, Fengjuan Huang ${ }^{1}$, Yiteng Liao ${ }^{1}$, Yiyang Liu ${ }^{3}$, \\ Jun Li ${ }^{1}$, Chunxia Chen ${ }^{1}$ and Xiao-Ping Chen ${ }^{1 *}$
}

\begin{abstract}
Background: Schistosomiasis is a chronic infection, where the host immune response to the parasite changes from a predominantly Th1 to Th2 phenotype, when parasite enters the egg stage, restraining the host inflammatory immune responses to achieve a longer survival in the host. On the other hand, the development of Th2 responses causes immunopathological changes such as liver fibrosis. Therefore identification of schistosome-derived Th2 inducing molecules is important in the understanding of pathogenesis of schistosomiasis. A cyclophilin A homologue of Schistosoma japonicum was reported to be an egg-stage specific antigen, but its immunogenicity and immunoregulatory activities remain unknown.
\end{abstract}

Methods: We cloned and expressed the gene of cyclophilin A from Schistosoma japonicum (AY814078), named as SjCyP18 based on its molecular weight. The expression profiles in different stages of S. japonicum were examined by RT-PCR and immunofluorescence assay. The immunogenicity of SjCyP18 was measured by the presence of IgG in the sera from S. japonicum infected patients and animals, and the Th2-promting activities were examined by the subclass of immunoglobulins against SjCyP18 and by the IL-4 induction in T cells following footpad injection of SjCyP18.

Results: The cloned SjCyP18 has 65\% homology with human or mouse cyclophilin A at the amino acid level. In contrast to reports as an egg-stage specific antigen, the gene was found to be expressed in all stages of $S$. japonicum. IgG responses against SjCyP18 were found in some S. japonicum infected patients and were significantly induced when infection become patent and produce eggs in infected mice. Furthermore, the Th2-promoting subclass of IgG1 was the predominant isotype in S. japonicum infected mice. More importantly, footpad injection of SjCyP18 induced a greater production of IL-4 than that of IFN- $\gamma$ by lymphocytes compared to responses from PBS injection controls.

Conclusion: The cyclophilin A homologue found in S. japonicum is immunogenic and promotes Th2 responses in vivo which may contribute to the establishment of chronic infection by schistosomes.

Keywords: Th2 response, Schistosoma japonicum, Cyclophilin A

\section{Background}

Schistosomiasis is endemic throughout the tropics and subtropics. Infection by Schistosoma japonicum remains an important public health burden in South and East Asia despite the efforts of ongoing control programmes $[1,2]$. In contrast to many other infectious organisms, schistosome parasites are able to survive in the infected host for years, or even decades. This worm has developed effective strategies to express molecules homologous to

\footnotetext{
*Correspondence: xc9718@hotmail.com

${ }^{\dagger}$ Equal contributors

'Department of Immunology, Tongji University School of Medicine, Shanghai 200092, China

Full list of author information is available at the end of the article
}

those from the host (molecular mimicry) to facilitate evasion of host immune surveillance. Indeed, recent studies on the transcriptome information from S. japonicum indicated that hundreds of parasite genes share similarities with host homologues [3,4].

The other common mechanism used by this worm to evade host immune defense is to modulate inflammatory immune responses to facilitate the establishment of chronic infection in the host. The immunomodulatory cytokine IL-10, for example, can be induced by cells of both innate and adaptive immunity including regulatory $\mathrm{T}$ cells in response to egg- or worm-derived antigens [5]. Ablation of IL-10 resulted in exaggerated granulomatous disease and

\section{Biomed Central}

(c) 2013 Li et al.; licensee BioMed Central Ltd. This is an open access article distributed under the terms of the Creative Commons Attribution License (http://creativecommons.org/licenses/by/2.0), which permits unrestricted use, distribution, and reproduction in any medium, provided the original work is properly cited. 
decreased host survival [6]. More importantly, the welldescribed IL-4-producing Th2 responses, induced when schistosome infection becomes patent and produces the egg-stage, contribute directly to the limitation of host proinflammatory reactions and the establishment of chronic infection. Examination of schistosome infection in mice defective for Th2 associated responses has shown that the inability to develop Th2 responses to restrain the initial pro-inflammatory response exacerbates acute schistosomiasis leading to earlier death $[7,8]$. Therefore, the Th2 response induced by schistosome infection is essential for the survival of both the host and the helminth worms with the cost of chronic schistisomiasis characterized by liver granulomatous inflammation and liver fibrosis.

It has been well established that egg antigens are primarily responsible for the Th2 induction [7]. The egg-derived IPSE/alpha-1 and omega-1, which are both glycoproteins secreted by the eggs have recently been shown to be involved in the initiation or potentiation of Th2 development in $S$. mansoni infection. IPSE/alpha-1 is able to trigger basophils to produce IL-4, which may contribute to Th2 polarization [9]. Omega-1, on the other hand, potently instructs DCs to prime highly Th2-polarized responses [10]. However, neither of these two molecules are fully responsible for the Th2 induction activities by soluble egg antigen (SEA) [10]. Therefore, more SEA-associated Th2-inducing molecules await characterization. In terms of $S$. japonicum, most of the identified molecules induce IFN- $\gamma$-dominant Th1 responses [11,12], except SjEsRRBL1, which is reported to elicit a mixed Th1/Th2 response [13]. The IPSE homologue cloned from S. japonicum failed to demonstrate stimulation of IL-4 production by basophils and no Omega-1 homologue was readily identified in $S$. japonicum (data not shown). Thus, the Th2-inducing molecules from $S$. japonicum are not well known.

In order to identify Th2-promoting molecules from $S$. japonicum, we focused on antigens reported to be highly or uniquely expressed in the eggs. The gene AY814078 was originally identified via high throughput sequencing as showing $65 \%$ homology with human cyclophilin A (HsCyPA)( AAH05982) [3] and reported to be egg-stage specific. Known as the target for the immunosuppressive drug cyclosporin A [14], cyclophilin A has been shown to have other effects. For example, cyclophilin A was reported to achieve anti-inflammatory effects both in vivo and in vitro via interacting with the membrane protein CD147 [15]. Furthermore, cyclosporin A demonstrated antimicrobial activity against a variety of eukaryotic pathogens including anti-schistosome effects [16]. The signature region making up the putative cyclosporin A-binding pocket is well conserved among cyclophilin A molecules from different organisms [17]. Although a number of cyclophilins have been uncovered in $S$. mansoni and S. japonicum [18-21], whether or not they are fully responsible for the anti-helminth activities by cyclosporin A remain unknown. Equally unknown are the immunoregulatory activities of these schistosome cyclophilins.

In this study, we cloned and expressed the gene AY814078, characterized its expression in different stages of worm development and analyzed its immunogenicity in infected mice and humans and its ability to induce Th2-polarized responses in mice. We found that cyclophilin A in S. japonicum ( $\mathrm{SjCyP18)}$ is a homologue of human $\mathrm{HsCyP} 18$, which is highly expressed in adult worms, eggs and in soluble egg antigens. Furthermore, footpad injection of SjCyP18 induced IL-4 production by activated lymphocytes isolated from draining lymph nodes, corresponding to a robust Th2-promoted IgG1 subclass found in S. japonicum infected mice.

\section{Methods}

\section{Animals and infection}

6-8 weeks old female C57BL/6 mice were purchased from SLAC Lab Animals CO (Shanghai). All mice were maintained under specific pathogen-free conditions and fed with standard laboratory food and water. All procedures performed on animals within this study were conducted in accordance with and by approval of the Internal Review Board of Tongji University School of Medicine. Mice were challenged by skin-penetrating infection of 20 cercaria of S. japonicum, which were shed from infected Oncomelania hupensis snails provided by National Institute of Parasitic Diseases at Shanghai, China. The animal protocol was approved by the Committee on the Ethics of Animal Experiments of Tongji University (Permit Number: 2009-0022). The human study was approved by the Ethics Committee of Tongji University.

\section{Preparation of S. japonicum samples}

The adult schistosomes were collected by perfusion from the portal vein of mice 42 days after infection. The soluble egg antigens (SEA) were made by the method developed by Brindley with modifications [22]. Livers from S. japonicum infected mice or livers from infected rabbits which were kindly provided by National Institute of Parasitic Diseases at Shanghai, China were chopped and homogenized in cold phosphate-buffered saline (PBS). $1 \mathrm{mg} / \mathrm{ml}$ collagenase IV, $10 \mu \mathrm{g}$ penicillin and $10 \mu \mathrm{g}$ streptomycin were added to the liver homogenate followed by incubation with shaking at $37^{\circ} \mathrm{C}$ overnight. The homogenate was centrifuged at $400 \mathrm{~g}$ for $5 \mathrm{~min}$ and the pellet resuspended in $3 \mathrm{ml}$ of PBS and applied to the top of a Percoll column prepared by mixing $8 \mathrm{ml}$ of Percoll with $32 \mathrm{ml}$ of $0.25 \mathrm{M}$ sucrose. The schistosome eggs were enriched in the pellet after centrifugation at $800 \mathrm{~g}$ for $10 \mathrm{~min}$. The purified eggs were washed 3 times in PBS containing $1 \mathrm{mM}$ EDTA and 
$1 \mathrm{mM}$ EGTA at $30 \mathrm{~g}$. Further purification of eggs was achieved by resuspension of the eggs in $0.5 \mathrm{ml}$ of PBS and re-application on to a Percoll column prepared by mixing $2.5 \mathrm{ml}$ of Percoll with $8.5 \mathrm{ml}$ of $0.2 \mathrm{M}$ sucrose and was pelleted and washed as before. Purified eggs were ultrasonicated for $10 \mathrm{~s}$ with an interval of $10 \mathrm{~s}$ for $10 \mathrm{~min}$ in $5 \mathrm{ml}$ PBS, and final soluble egg antigens (SEA) were obtained from the supernatants after being centrifuged at $400 \mathrm{~g}$ for $15 \mathrm{~min}$ and were filtered through $0.22 \mu \mathrm{m}$ filters.

\section{Cloning, expression and purification of recombinant SjCyP18 and HsCyP18 and generation of anti- SjCyP18 mouse serum}

Total RNA was isolated from eggs with TRIZOL reagent (Invitrogen) according to the manufacturer's instructions. First strand cDNA was synthesized using reverse transcriptase AMV and oligo ( $\mathrm{dT}$ ) primer (Invitrogen) as described below. The primers were designed based on the posted sequence from AY814078 as sense primers 5' GCCGGATCCTCAACGTTTCCCAGGGT-3' containing BamHI and ansisense primers 5'-GTCCTCGAGTTAGC ATTGCCCGCAATTAAC-3' containing Xhol. The SjCyP18 gene was obtained by PCR reaction following the amplification conditions as below, and a recombinant pET28a$\mathrm{SjCyP} 18$ vector was constructed after enzyme cutting and ligation. Secreted products in the supernatants were collected from BL21 (Incubation for $1.5 \mathrm{~h}$ at $37^{\circ} \mathrm{C}$ until the OD600 reached 0.6, IPTG induction at $0.5 \mathrm{mmol} / \mathrm{L}$ at $37^{\circ} \mathrm{C}$ for $4 \mathrm{~h}$ ) and were purified with Ni-containing column (His60 Ni Superflow Resin, Takara) and concentrated by Ultracel PL-10 (Millipore). The polymyxin B-Agarose (Sigma) treatment was applied to remove endotoxin. Purified protein was run on SDS-PAGE and analyzed with either Coomassie blue or western blot.

Antisera to $\mathrm{SjCyP} 18$ were raised in mice by injection of mice with $50 \mu \mathrm{g}$ of purified recombinant $\mathrm{SjCyP} 18$ protein subcutaneously in the presence of complete Freund's adjuvant CFA (Sigma) in $100 \mu$ l. Boosting injection was administered by $100 \mu \mathrm{l}$ incomplete Freund's adjuvant IFA with $25 \mu \mathrm{g}$ recombinant $\mathrm{SjCyP18}$ protein at day 14 and day 28 respectively. The serum obtained at day 42 was used in the study for western blot or immunofluorescence staining analysis.

\section{Detection of SjCyP18 by RT-PCR}

Total RNA from cercariae, adult male and female worms and eggs was isolated by TRIzol (Invitrogen) according to the manufacturer's instructions. $1 \mu \mathrm{g}$ of RNA was reverse transcribed into cDNA with oligo dT primers in $20 \mu \mathrm{l}$ containing $5 \times \mathrm{RT}$ buffer $(4 \mu \mathrm{l}), 10 \mathrm{mM}$ dNTP $(1 \mu \mathrm{l}), 10 \mu \mathrm{M}$ Oliga dT $(0.5 \mu \mathrm{l})$, RNase inhibitor (40 units) and AMV (5 units) at $42^{\circ} \mathrm{C}$ for $1 \mathrm{~h}$. To exclude the possibility of genomic DNA contamination in RNA preparations, a parallel reaction without reverse transcriptase was performed. $2 \mu \mathrm{l} \mathrm{cDNA}$ or $2 \mu \mathrm{l}$ RNA was used for PCR which was performed in a volume of $20 \mu \mathrm{l}$ containing $2 \times$ EasyTaq $^{\circ}$ PCR SuperMix (10 $\mu$ l,TransGen Biotech), $20 \mu \mathrm{M}$ primer $\left(0.5 \mu \mathrm{l}\right.$ for each) with reaction as $94^{\circ} \mathrm{C}$ 5 min followed by $94^{\circ} \mathrm{C} 30 \mathrm{sec}, 55^{\circ} \mathrm{C} 30 \mathrm{sec}$ and $72^{\circ} \mathrm{C}$ $1 \mathrm{~min}$ for 30 cycles with final extension of $72^{\circ} \mathrm{C}$ for $5 \mathrm{~min}$. The sequences of primers for PCR were the same as those for cloning primers.

\section{Detection of SjCyP18 by immunofluorescence staining}

Polyclonal antibodies for $\mathrm{SjCyP18}$ were raised in-house and used in diluted mouse serum (1:50 in TBST). Mouse antiserum against $S$. japonicum tetraspanins anti-TSP2 (1:50 dilution, provided by Institute of Infectious Disease and Vaccine Development, Tongji University) [23] was used as a positive control for immunostaining. The secondary antibody used was AlexaFluor 488-conjugated goat anti-mouse IgG (1:250, Invitrogen). The eggs, adult male and female worms and cercariae of $S$. japonicum were fixed in $10 \%$ buffered formalin for $10 \mathrm{~min}$. The fixed organisms were frozen in OCT medium (Tissue-Tek) and stored at $-80^{\circ} \mathrm{C}$ first and were then sectioned and mounted on glass slides coated with gelatin. Each section was cut to $10 \mu \mathrm{m}$ thickness with Cryostat(Microm HM525) at $-20^{\circ} \mathrm{C}$. After being blocked with $10 \%$ bovine serum-containing medium for $1 \mathrm{~h}$ at room temperature, slides were dried and then washed three times for $2 \mathrm{~min}$ interval with PBST (PBS, pH 7.4 with $0.1 \%$ Tween-20). Slides were incubated with primary antibodies overnight at $4^{\circ} \mathrm{C}$ in PBS. After being washed in PBS, slides were incubated with secondary antibodies for $10 \mathrm{~min}$ at room temperature in the dark. The slides were mounted with Antifade Mounting Medium (Beyotime Institute of Biotechnology) after final wash with PBS. In addition to being stained by anti-SjCyP18, sections were also stained by either anti-SjTSP2-serum or normal mouse serum ( 5 normal sera were pooled) for positive and negative controls, respectively. Images were collected on an inverted fluorescence microscope. Staining was also carried out on fixed, whole-mount cercariae.

\section{Measurement of antibodies against SjCyP18 or against SEA in patients and infected mice by ELISA}

Mouse sera were collected six weeks or at indicated infected time points after S. japonicum infection. ELISA was performed to determine antigen-specific total IgG, IgG1 and IgG2c. The 96-well microtiter plates were coated with either purified SjCyP18 at $5 \mu \mathrm{g} / \mathrm{ml}$ or SEA at $10 \mu \mathrm{g} / \mathrm{ml}$ in $50 \mathrm{mM}$ carbonate buffer (pH9.6) overnight at $4^{\circ} \mathrm{C}$, washed five times with PBST and blocked with $5 \%$ nonfat dry milk in PBST at room temperature for $1 \mathrm{~h}$. After being washed five times, the wells were incubated with infected mouse serum at 1:200 dilutions at $37^{\circ} \mathrm{C}$ for $2 \mathrm{~h}$. The plates were washed with PBST three 
times, followed by adding peroxidase-conjugated antimouse IgG at 1:2000 or anti-mouse IgG1 or anti-mouse IgG2c at 1:7500 (Santa Cruz) at $50 \mu \mathrm{l} /$ well. After $2 \mathrm{~h}$ incubation at room temperature and wash, the final color development was achieved by adding peroxidase substrate ABTS (2,2' -Azino-bis(3-Ethylbenzthiazoline-6-Sulfonic Acid) (Sigma) to each well at $100 \mu \mathrm{l} /$ well and absorbance was measured at $405 \mathrm{~nm}$.

A similar method was used to measure the antiSjCyP18 IgG present in sera from 5 normal human subjects and 10 patients who were positive for eggs in the feces by the Kato-Katz test. The sera were provided by Institute of Infectious Disease and Vaccine Development, Tongji University and were used at a dilution of 1:400. The detection was made by peroxidase-conjugated antihuman IgG (Promega) diluted at 1:2500.

\section{Induction of Th2 response in vivo}

Mice were immunized subcutaneously in the hind footpad with $20 \mu \mathrm{g}$ SEA or $15 \mu \mathrm{g} \mathrm{SjCyP18}$ in a volume of $20 \mu \mathrm{l}$ in PBS and the draining popliteal lymph nodes were removed aseptically from 4d immunized or naive mice. Lymphocyte suspensions were produced by passing through a $200 \mu \mathrm{m}$ mesh and were stimulated by anti-CD3 and anti-CD28 antibodies (eBioscience) for 48 hours and the concentration of IL-4 and IFN- $\gamma$ in the supernatants were analyzed as described [24].

\section{Statistical analysis}

Unpaired Student's $\mathrm{t}$ test and one-way or two-way ANOVA were used to determine the statistical significance of the differences among groups. Each individual experiment was conducted with at least 3 mice and each experiment was repeated at least twice.

\section{Results}

\section{Cloning, expression and purification of SjCyP18}

Belonging to the immunophilin family, cyclophilins are known to have important intracellular regulatory activities in T-cell activation. Intrigued by its high homology with $\mathrm{HsCyP} 18$ and the reported egg-stage-specific expression of AY814078 from S. japonicum [3], we cloned this gene from egg cDNA. The successfully cloned fulllength cDNA contained 495 bp encoding an ORF of 164 amino acids with a theoretical molecular mass of $18 \mathrm{kDa}$, thus named as $\mathrm{SjCyP18}$. Amino acid sequence alignment analysis showed that $\mathrm{SjCyP} 18$ is $66 \%$ identical to the human CyP18 (GenBank:AAH05982) or mouse CyP18 (GenBank:P17742) and 62\% identical to S. mansoniderived cyclophilin A SmCyP18 (GenBank:AAC47317) [19]. Comparing to a previously identified S. japonicum-derived cyclophilin A (GenBank:AAA29863) [18], $60 \%$ similarity was observed. Furthermore, a newly described S. japonicum-derived protein whose gene sequence is 99\% identical to our AY814078 gene, was also characterized as S. japonicum cyclopilin A (GenBank: FN323717) [25].

To further study the function of SjCyP18, the prokaryotic expression vector pET28a-SjCyP18 was constructed and His-SjCyP18 fusion protein was abundantly induced and purified. The successful purification of $\mathrm{SjCyP} 18$ was verified by western blot either by anti-His antibodies or by polyclonal antibodies generated against SjCyP18 (data not shown).

\section{Analysis of expression of SjCyP18 on schistosomes}

Unlike the reported result that $\mathrm{SjCyP} 18$ expression is egg-stage specific [3], our RT-PCR results demonstrated that this gene was expressed in all schistosome life cycle stages examined (cercariae, adults and eggs) (Figure 1A). We then applied immunofluorescence staining to observe the expression and localization of $\mathrm{SjCyP} 18$ in schistosomes in different life stages with mouse antiserum prepared against recombinant $\mathrm{SjCyP18}$. The results showed that immunostaining were found in cercariae, female and male worms and eggs. The positive staining disappeared after preincubation of antiserum with recombinant SjCyP18 indicating that the positive immunostaining signals were specific (data not shown). Similar to the outer tegument staining pattern of TSP2 on adult $S$. masoni [26] and S.japonicum [23], the positive staining for $\mathrm{SjCyP} 18$ was also found at the surfaces of both female and male adult worms, except that the reproductive tissues in female worms was only positive for anti-TSP2 (Figure 2B).

\section{Anti-SjCyP18 lgG level is increased in S. japonicum infected patients}

Due to similar surface expression pattern between SjCyP18 and TSP2 which indicates that SjCyP18 may also function as an immunogen, such as TSP2 [26], specific anti-SjCyP18 IgG was measured in serum samples from $S$. japonicum infected patients who were all positive for eggs in the feces by the Kato-Katz test. Similar to the increased levels of anti-SEA IgG in patients, the average level of anti-SjCyP18 IgG was also significantly higher in patients than that of normal sera (Figure 2A), suggesting that SjCyP18 is immunogenic during S. japonicum infection.

\section{Th2-associated IgG1 is predominantly induced in S. japonicum infected mice}

When S. japonicum infected mice sera were examined, the levels of anti-SjCyP18 IgG were increased significantly between days 35 to 42 post-infection compared to those at 14 days after infection and in uninfected mice (Figure 2B). 


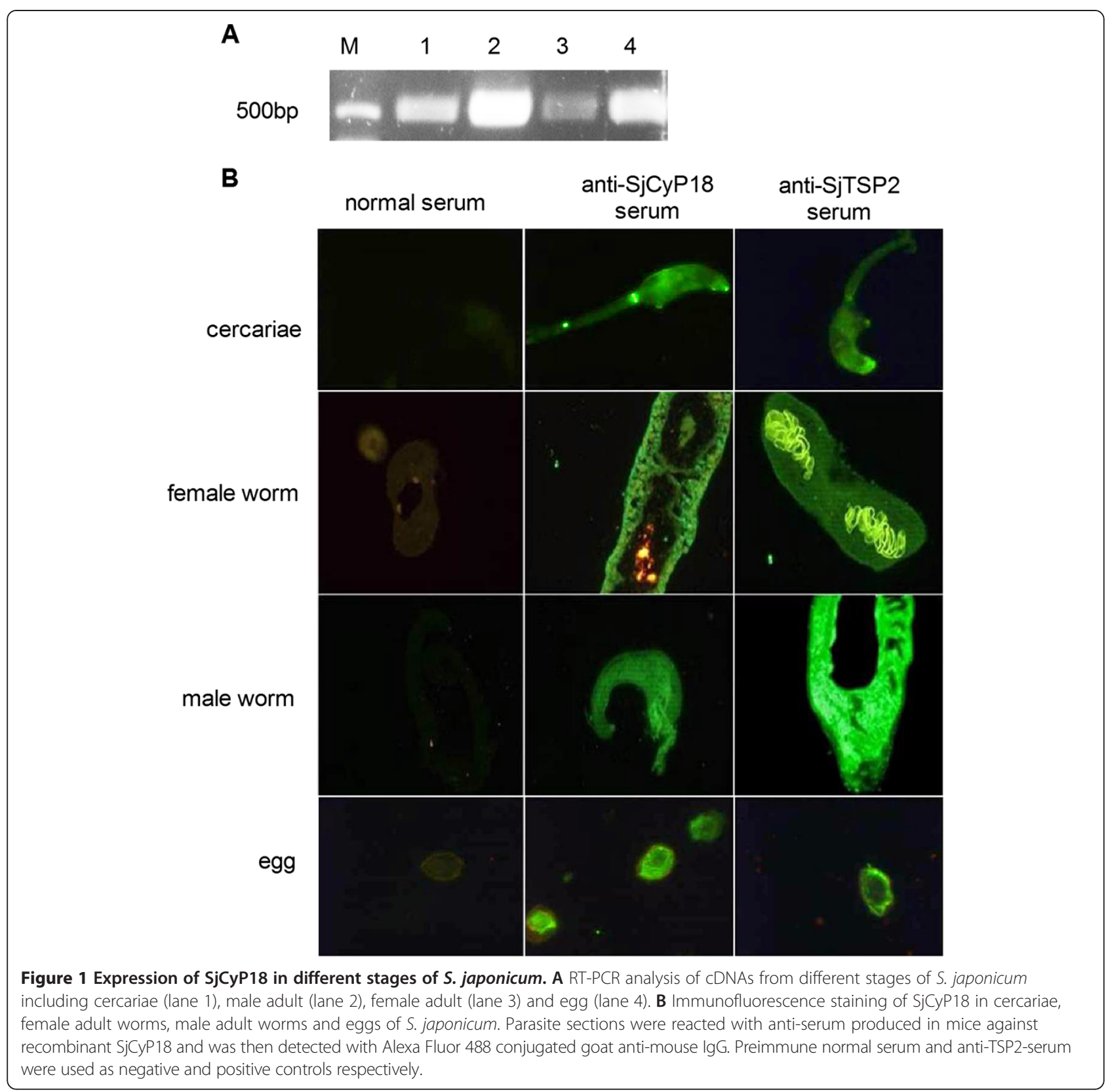

Furthermore, we tested the subclasses of the SjCyP18specific IgG and found that Th2-associated IgG1 levels were higher than Th1-associated IgG2c levels, corresponding to the dominant IgG1 subclass found in infected mice when SEA was analysed (Figure 2C). These may indicate that SjCyP18 could contribute to induce a Th2-dominated immune response during post patent $S$. japonicum infection.

\section{Induction of Th2 response by SjCyP18 in vivo}

Since SjCyP18 appears to preferentially induce an IgG1dominant response, its capacity to promote IL-4- production was then examined. When SEA was injected via footpad, lymphocytes from popliteal draining lymph nodes were found to produce large quantities of IL-4 following restimulation of lymphocytes with anti-CD3 and anti-CD28 for 48 hours in vitro, although IFN- $\gamma$ production was also induced compared to PBS controls. When SjCyP18 was injected, IL-4 production was also significantly induced comparing to PBS control although at much lower levels than that induced by SEA injection. However, no enhanced induction of IFN- $\gamma$ was observed comparing to PBS treatment (Figure 3). Therefore, SjCyP18 was able to promote a Th2 response in vivo. 

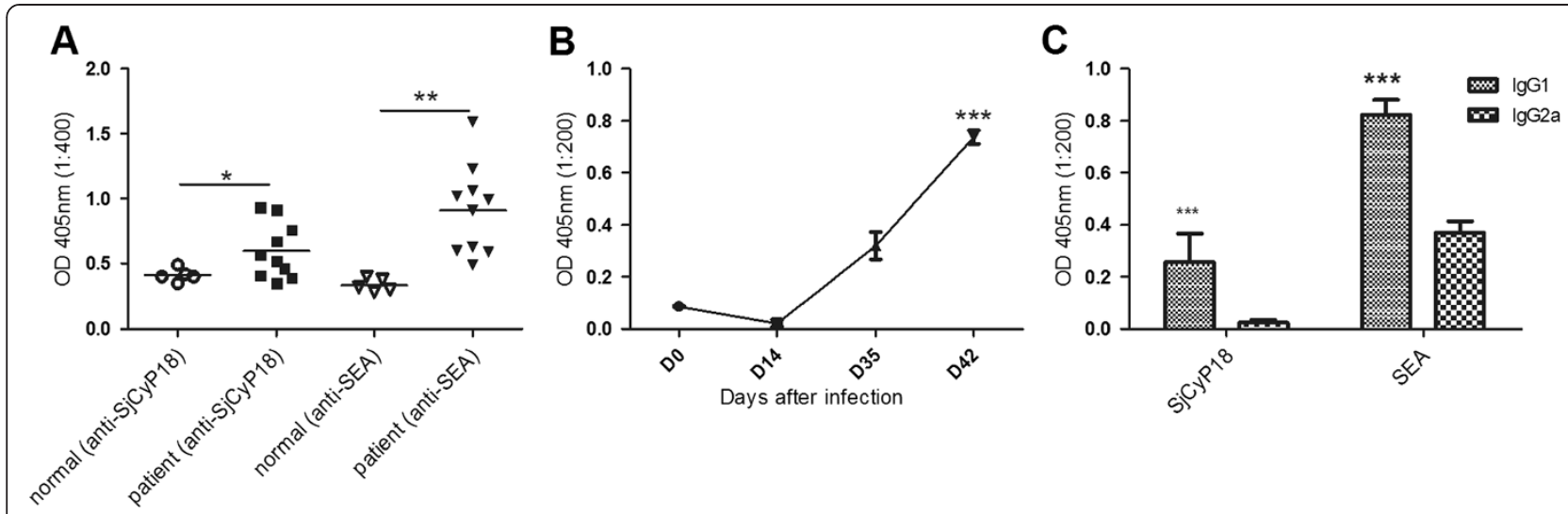

Figure 2 Analysis of anti-SjCyP18 IgG in S. japonicum infected patients and anti-SjCyP18 IgG and its subclasses in infected mice. A Sera from 10 S. japonicum infected patients who were positive by Kato-Katz test and from 5 normal subjects were measured for anti-SjCyP18 or anti-SEA IgG by ELISA. The significance test was analyzed by unpaired t-test with * as $p<0.05$ and ${ }^{* *}$ as $p<0.01$. B The time course of antiSjCyP18 IgG in S. japonicum infected mice, showing the mean \pm SEM from 4 mice, representative of two separate experiments. One-way ANOVA was used to test the significance on the levels of day 42 with all the other time points with *** as $p<0.001$. C Mouse sera from $42 \mathrm{~d}$ Sj infected mice were measured for anti-SjCyP18 or anti-SEA IgG1 or IgG2C by ELISA as described in the methods. The results shown on IgG subclasses were the mean value of 4 mice and a representative of 5 separately performed experiments. Two-way ANOVA was used to test the significances of differences with *** as $p<0.001$.

\section{Discussion}

Multiple cyclophilin isoforms have been reported in mammalian cells including HsCyPA also called HsCyP18, HsCyP40, $\mathrm{HsCyPB}, \mathrm{HsCyPC}, \mathrm{HsCyPD}, \mathrm{HsCyPE}, \mathrm{HsCyPNK}$ and others. $\mathrm{HsCyPA}$ and $\mathrm{HsCyP} 40$ are abundantly expressed in the cytosol in various types of cells; others may possess signal sequences that target them to specific organelles like ER and mitochondria [27]. As a prototypical minimal cyclophilin, which contains just the peptidyl-prolyl cis-trans isomerase (PPIase) domain and is the target for immunosuppressant drug cyclosporin A, CyP18 have also been described in prokaryocytes and eukaryotic pathogens like schistosomes and Plasmodium falciparum [28]. In this study, we reported a HsCyP18 homologue in Schistosoma japonicum and it is found to be expressed at all stages of life cycles in schistosomes and abundantly present in soluble egg antigens.

The activities of the widely expressed CyPA in mammalian and nonmamalian cells have not been definitely clarified except for its well-known role as a receptor for the immunosuppressive drug. A recent study on Leishmania donovani has suggested that CyPA played essential roles in proliferation and survival of the organism, which is independent of its PPIase activity [29]. On the other hand, several studies reported that CyPA can be secreted from the cells following inflammatory stimulation or oxygen stress [30]. Secreted CyPA can in turn enhance the adhesion activities, ROS production and proinflammatory activities on endothelial cells which is probably involved in the pathogenesis of arthrosclerosis
[31,32]. Furthermore, CyPA possesses chemoattractant activities on neutrophils, eosinophils, monocytes and $\mathrm{T}$ cells [33], relating to the development of rheumatoid arthritis $[34,35]$. The recombinant SjCyP18 in this study failed to display chemotactic activities on human monocytes (data not shown).

Our studies, however, did demonstrate that the schistosome-derived CyPA played a role in the interplay between worms and host. It was an immunogen that was able to induce IgG production especially IgG1 in infected mice, corresponding to its capacity to promote a Th2 response when injected in vivo. Th2 responses are known to be a critical response for the helminth to subvert the host immune response create a co-existing state [36]. The responsible molecules present in schistosomes especially in SEA for the induction of Th2 response are not completely elucidated. In $S$. mansoni infection, omega-1 was largely responsible for the Th2polarization by SEA in vitro, but in vivo experiments demonstrated that its role was limited [10]. To date, there is no omega-1 homologue reported in S. japonicum and sequence alignment analysis performed by us failed to find any significant homologue molecule in $S$. japonicum either (data not shown). Therefore, SjCyP18 is the first molecule reported from S. japonicum, which was able to induce a mild Th2 response in vivo independently. Moreover, we have reason to predict that there should exist other potent molecules in the SEA from $S$. japonicum, since the IL-4-promoting activities induced by $\mathrm{SjCyP} 18$ was much weaker than induced by SEA. 


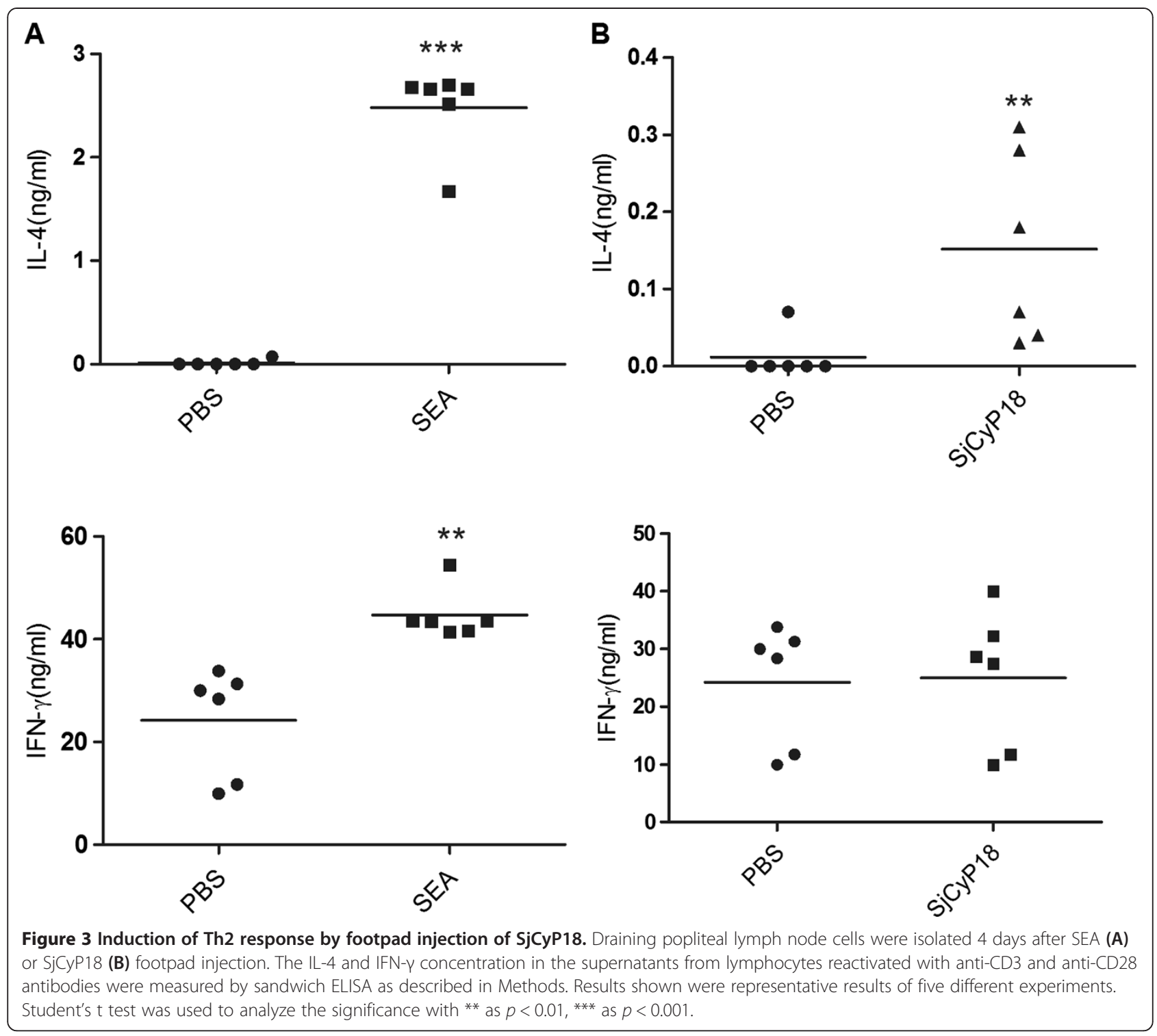

The potential of SjCyP18 as a vaccine candidate was also examined when the molecule was injected with or without CFA prior to S. japonicum infection. After three immunizations of SjCyP18, significant amount of anti-SjCyP18 IgG was induced (data now shown). Although a recent study declared that a $S$. japonicum cyclophilin A homologue protein could reduce the worm burden and liver egg burden in S. japonicum challenged mice [25], no significant protective effect was observed in our study (data not shown). The lack of protective effects by $\mathrm{SjCyP} 18$ immunization may be associated with the Th2-inducing ability of this molecule. Usually a Th1-dominant response is associated with protective effects in the $S$. japonicum infection model [37].

\section{Conclusions}

In summary, we cloned and expressed the gene of $S$. japonicum-derived cyclophilin A, SjCyP18, which has about 66\% homology with human CyP18 at the amino acid level. Furthermore, SjCyP18 was expressed by all stages of S. japonicum and was localized to the outer tegument of adult worms. IgGs against $\mathrm{SjCyP} 18$ were found in chronically infected patients as well as in mice during post-patent infections. In vivo injection of SjCyP18 facilitated an IL-4-producing Th2 response, corresponding to the dominant IgG1 response seen in infected mice. Altogether, our study demonstrates another important role played by a $S$. japonicum-derived cyclophilin in the interplay between host and pathogens. 


\section{Abbreviations}

SEA: Soluble egg antigen; CyPA: Cyclophilin A; HsCyPA: Human cyclophilin A; Sj: Schistosoma japonicum.

\section{Competing interests}

The authors declare that they have no competing interests.

\section{Authors' contributions}

$J \mathrm{~L}, \mathrm{WZ}, \mathrm{WS}$ and XPC participated in the design of the study. $J \mathrm{~L}, \mathrm{XC}$ and WS did the experiments on cloning, expression and serum response on SjCyP18. WZ and LC did the Th2 induction experiments. YL and YL cloned and expressed the HsCyP18. JL, WZ and XPC wrote the manuscript. All authors read and approved the final manuscript.

\section{Acknowledgements}

This work was supported by NSFC(no.81071382)and National Basic Research Program (973 program) (no. 2007CB513100) from the Chinese government. Thanks for the human sera generously provided by Dr. Weiqing Pan from the Institute of Infectious Disease and Vaccine Development, Tongji University. Thanks for the technical help provided by Jun Li and Yaoming He.

\section{Author details}

'Department of Immunology, Tongji University School of Medicine, Shanghai 200092, China. ${ }^{2}$ Department of Pathogen Biology, Tongii University School of Medicine, Shanghai 200092, China. ${ }^{3}$ Shanghai Institute of Pharmaceutical Industry, Shanghai 200040, China.

Received: 26 June 2013 Accepted: 14 November 2013

Published: 17 November 2013

\section{References}

1. Lustigman S, Geldhof P, Grant WN, Osei-Atweneboana MY, Sripa B, Basanez MG: A research agenda for helminth diseases of humans: basic research and enabling technologies to support control and elimination of helminthiases. PLoS Negl Trop Dis 2012, 6:e1445.

2. Utzinger J, Zhou XN, Chen MG, Bergquist R: Conquering schistosomiasis in China: the long march. Acta Trop 2005, 96:69-96.

3. Hu W, Yan Q, Shen DK, Liu F, Zhu ZD, Song HD, Xu XR, Wang ZJ, Rong YP, Zeng LC, et al: Evolutionary and biomedical implications of a Schistosoma japonicum complementary DNA resource. Nat Genet 2003, 35:139-147.

4. Liu F, Hu W, Cui SJ, Chi M, Fang CY, Wang ZQ, Yang PY, Han ZG: Insight into the host-parasite interplay by proteomic study of host proteins copurified with the human parasite, Schistosoma japonicum. Proteomics 2007, 7:450-462

5. Hesse MPC, Belkaid Y, Prufer J, Mentink-Kane M, Leusink M, Cheever AW, Shevach EM, Wynn TA: The pathogenesis of schistosomiasis is controlled by cooperating IL-10-producing innate effector and regulatory $T$ cells. J Immunol 2004, 172:3157-3166.

6. Hoffmann KF, Cheever AW, Wynn TA: IL-10 and the dangers of immune polarization: excessive type 1 and type 2 cytokine responses induce distinct forms of lethal immunopathology in murine schistosomiasis. $\mathrm{J}$ Immunol 2000, 164:6406-6416.

7. Grzych JM, Pearce E, Cheever A, Caulada ZA, Caspar P, Heiny S, Lewis F, Sher A: Egg deposition is the major stimulus for the production of Th2 cytokines in murine schistosomiasis mansoni. J Immunol 1991, 146:1322-1327.

8. Brunet LR, Finkelman FD, Cheever AW, Kopf MA, Pearce EJ: IL-4 protects against TNF-alpha-mediated cachexia and death during acute schistosomiasis. J Immunol 1997, 159:777-785.

9. Schramm G, Falcone FH, Gronow A, Haisch K, Mamat U, Doenhoff MJ, Oliveira G, Galle J, Dahinden CA, Haas H: Molecular characterization of an interleukin-4-inducing factor from Schistosoma mansoni eggs. J Biol Chem 2003, 278:18384-18392.

10. Everts B, Perona-Wright $\mathrm{G}$, Smits $\mathrm{HH}$, Hokke $\mathrm{CH}$, van der Ham AJ, Fitzsimmons $\mathrm{CM}$, Doenhoff MJ, van der Bosch J, Mohrs K, Haas H, et al: Omega-1, a glycoprotein secreted by Schistosoma mansoni eggs, drives Th2 responses. J Exp Med 2009, 206:1673-1680.

11. Qiu C, Liu S, Hong Y, Fu Z, Wei M, Ai D, Lin J: Molecular characterization of thyroid hormone receptor beta from Schistosoma japonicum and assessment of its potential as a vaccine candidate antigen against schistosomiasis in BALB/c mice. Parasit Vectors 2012, 5:172.
12. He S, Yang L, Lv Z, Hu W, Cao J, Wei J, Sun X, Yang J, Zheng H, Wu Z: Molecular and functional characterization of a mortalin-like protein from Schistosoma japonicum (SjMLP/hsp70) as a member of the HSP70 family. Parasitol Res 2010, 107:955-966.

13. Wu X, Zhao B, Hong Y, Li X, Peng J, Zhang J, Wang F, Shi Y, Fu Z, Lin J: Characterization of Schistosoma japonicum estrogen-related receptor beta like 1 and immunogenicity analysis of the recombinant protein. Exp Parasitol 2012, 131:383-392.

14. Emmel EA, Verweij CL, Durand DB, Higgins KM, Lacy E, Crabtree GR: Cyclosporin A specifically inhibits function of nuclear proteins involved in T cell activation. Science 1989, 246:1617-1620.

15. Yurchenko V, Constant S, Eisenmesser E, Bukrinsky M: Cyclophilin-CD147 interactions: a new target for anti-inflammatory therapeutics. Clin Exp Immunol 2010, 160:305-317.

16. Bout D, Deslee D, Capron A: Antischistosomal effect of cyclosporin A: cure and prevention of mouse and rat schistosomiasis mansoni. Infect Immun $1986,52: 823-827$

17. Pflugl G, Kallen J, Schirmer T, Jansonius JN, Zurini MG, Walkinshaw MD: X-ray structure of a decameric cyclophilin-cyclosporin crystal complex. Nature 1993, 361:91-94.

18. Argaet VP, Mitchell GF: Cyclophilin of Schistosoma japonicum. J Parasitol 1992, 78:660-664.

19. Klinkert MQ, Bugli F, Cruz J, Engels B, Cioli D: Sequence conservation of schistosome cyclophilins. Mol Biochem Parasitol 1996, 81:239-242.

20. Kiang D, El Ghazalie NE, Medhat AM, Abdel-Fattah M, Karim AM, LoVerde PT: Identification and characterization of Schistosoma mansoni p17.7, a cyclophilin. Mol Biochem Parasitol 1996, 76:73-82.

21. Peng J, Han H, Hong Y, Wang Y, Guo F, Shi Y, Fu Z, Liu J, Cheng G, Lin J: [Cloning and expressing of cyclophilin B gene from Schistosoma japonnicum and the analysis of immunoprotective effect]. Sheng Wu Gong Cheng Xue Bao 2010, 26:317-323.

22. Dalton JP, Day SR, Drew AC, Brindley PJ: A method for the isolation of schistosome eggs and miracidia free of contaminating host tissues. Parasitology 1997, 115(Pt 1):29-32.

23. Jiang $Y, X u$ X, Qing X, Pan W: Identification and characterization of six novel tetraspanins from Schistosoma japonicum. Parasit Vectors 2011, 4:190

24. He Y, Li J, Zhuang W, Yin L, Chen C, Li J, Chi F, Bai Y, Chen XP: The inhibitory effect against collagen-induced arthritis by Schistosoma japonicum infection is infection stage-dependent. BMC Immunol 2010, 11:28.

25. Han H, Peng J, Hong Y, Fu Z, Xu J, Lin J, Tao J: Molecular cloning and characterization of a cyclophilin A homologue from Schistosoma japonicum. Parasitol Res 2012, 111:807-817.

26. Tran MH, Pearson MS, Bethony JM, Smyth DJ, Jones MK, Duke M, Don TA, McManus DP, Correa-Oliveira R, Loukas A: Tetraspanins on the surface of Schistosoma mansoni are protective antigens against schistosomiasis. Nat Med 2006, 12:835-840.

27. Lee J, Kim SS: Current implications of cyclophilins in human cancers J Exp Clin Cancer Res 2010, 29:97.

28. Page AP, Kumar S, Carlow CK: Parasite cyclophilins and antiparasite activity of cyclosporin A. Parasitol Today 1995, 11:385-388.

29. Yau WL, Blisnick T, Taly JF, Helmer-Citterich M, Schiene-Fischer C, Leclercq O, Li J, Schmidt-Arras D, Morales MA, Notredame C, et al: Cyclosporin A treatment of Leishmania donovani reveals stage-specific functions of cyclophilins in parasite proliferation and viability. PLoS Negl Trop Dis 2010, 4:e729.

30. Kim SH, Lessner SM, Sakurai Y, Galis ZS: Cyclophilin A as a novel biphasic mediator of endothelial activation and dysfunction. Am J Pathol 2004, 164:1567-1574.

31. Jin ZG, Lungu AO, Xie L, Wang M, Wong C, Berk BC: Cyclophilin A is a proinflammatory cytokine that activates endothelial cells. Arterioscler Thromb Vasc Biol 2004, 24:1186-1191.

32. Suzuki J, Jin ZG, Meoli DF, Matoba T, Berk BC: Cyclophilin A is secreted by a vesicular pathway in vascular smooth muscle cells. Circ Res 2006, 98:811-817.

33. Arora K, Gwinn WM, Bower MA, Watson A, Okwumabua I, MacDonald HR, Bukrinsky MI, Constant SL: Extracellular cyclophilins contribute to the regulation of inflammatory responses. J Immunol 2005, 175:517-522.

34. Guo FJ, Wang Y, Li Y, Peng JB, Hong Y, Oiu CH, Chen S, Fu ZQ, Shi YJ, Lin JJ: Evaluation on the immununo-protective efficacy of the recombinant antigen SjPGAM-SjEnol against Schistosoma japonicum in mice. Zhongguo $\mathrm{Ji}$ Sheng Chong Xue Yu Ji Sheng Chong Bing Za Zhi 2010, 28:246-251. 
35. Yang Y, Lu N, Zhou J, Chen ZN, Zhu P: Cyclophilin A up-regulates MMP-9 expression and adhesion of monocytes/macrophages via CD147 signalling pathway in rheumatoid arthritis. Rheumatology (Oxford) 2008, 47:1299-1310.

36. Pearce EJ, MacDonald AS: The immunobiology of schistosomiasis. Nat Rev Immunol 2002, 2:499-511.

37. Xu X, Zhang D, Sun W, Zhang Q, Zhang J, Xue X, Shen L, Pan W: A Schistosoma japonicum chimeric protein with a novel adjuvant induced a polarized Th1 immune response and protection against liver egg burdens. BMC Infect Dis 2009, 9:54.

doi:10.1186/1756-3305-6-330

Cite this article as: Li et al:: Cyclophilin A from Schistosoma japonicum promotes a Th2 response in mice. Parasites \& Vectors 2013 6:330.

\section{Submit your next manuscript to BioMed Central and take full advantage of:}

- Convenient online submission

- Thorough peer review

- No space constraints or color figure charges

- Immediate publication on acceptance

- Inclusion in PubMed, CAS, Scopus and Google Scholar

- Research which is freely available for redistribution 